\title{
Input-to-state stability in the meaning of switching for delayed feedback switched stochastic financial system
}

\author{
Ruofeng Rao ${ }^{\mathrm{a}}$, \\ a Department of Mathematics, Chengdu Normal University, Chengdu , 611130, China
}

\begin{abstract}
Financial system is essentially chaotic and unstable if there is not any external inputs. By means of Lyapunov function method, design of switching law, novel fuzzy assumption, $L^{p}$ estimation technique and Laplace semigroup theory, the author presents the boundedness and LMI-based (globally) asymptotical input-to-state stability criteria of financial systems. Particularly, the globally asymptotical stability in the meaning of switching implies that when the time $t$ is big enough, the dynamic of any subsystem must approach its unique equilibrium point. Besides, the global financial crisis often erupts periodically, which illuminates that the global stability in the classical sense is actually meaningless. So the stability in the meaning of switching proposed in this paper is suitable and appropriate. Numerical examples illuminate the effectiveness of the obtained results.
\end{abstract}

Keywords: switched system; asymptotical stability; Lyapunov function; $L^{p}$ space; non-Lipschitz function

\section{Introduction: On the difficulties brought about by nonlinear system with non-Lipschitz functions}

In recent decades, a kind of complex financial system model consisting of production sub-blocks, currency subblocks, securities sub-blocks and labor sub-blocks has become a hot topic for many researchers because it well simulates the dynamic behavior of important economic indicators in the real financial market ([1-9] and the references therein), and a series of beneficial economic macro-control and stability analysis are obtained, which are conducive to macro-control and market self-regulation with theoretical guidance ([1-10]). But the previous research only involves the stability of the equilibrium point with a fixed interest rate, while the financial market is complex and changeable, and the market will adjust itself, and the financial management department will carry out macro-control according to the market situation. Its purpose is not necessarily to pursue the stability of the equilibrium point with a certain fixed interest rate, but to constantly consciously or unconsciously switch to equilibrium points of different interest rates (positive or negative or zero) to achieve the prosperity and stability of the financial market. Besides, the important economic indicators, such as savings, the unit investment cost, and the elasticity of commodity demand, has certain uncertainty. It is necessary to deal with the important economic index parameters obtained by previous statistics or different ways with fuzzy method. Takagi-Sugeno (T-S) fuzzy model will be considered in this paper, for

Email address: ruofengrao@163.com (Ruofeng Rao )

Preprint submitted to $* * * * * * * * *$

September 3, 2020 
Takagi-Sugeno fuzzy models have excellent ability in analyzing, synthesizing, and approximating complex dynamical behaviors by a set of IF-THEN rules ([14-18]). Thereby, switched models and fuzzy models are usually investigated in various control systems ([11-21]). Due to the random inflows and outputs of foreign capital will produce real-time or delayed disturbance to economic indicators, Ito stochastic models are often considered in the modeling of various control systems ([22-27]). In real financial markets, the government purchase will promote economic prosperity and stability, which will randomly improve some important economic indicators, such as savings, the unit investment cost, and represents the elasticity of commodity demand. Because of the lag of macro-control effect in the real market, the effect of macro-control is not obvious when the market is switched to one subsystem and only takes effect when the market is switched to another subsystem. Therefore, one of the main tasks of this paper is to design such a switching rule that the whole switching system is stable although some subsystems are unstable.

On the other hand, the financial system is the nonlinear system with non-Lipschitz functions, which brings about the true difficulties. That is, the globally asymptotical stability in the meaning of switching introduced by [32, Definition 3] may truly be not available for the financial system. In fact, according to [33,34], the unique existence of the solution of a system can be guaranteed if the active functions are Lipschitz continuous and growing linearly. However, the financial systems studied by this paper are complex and chaos due to the non-Lipschitz functions with superlinear growth. To overcome this difficulty, a suitable Lyapunov-Krasovskii function is given to create conditions making the switched financial system stable globally.

It is found from the study of financial mathematical model that the essence of financial system is chaotic and unstable. This is also in line with the unpredictability of the real financial market. In fact, the cyclical outbreak of the world economic crisis has confirmed this view. If the financial market is to be stable, the government must be active in taking remedial measures at a certain time. Thereby, input-to-state stability of switched financial system is studied in this paper. Due to the difficulties brought about by non-Lipschitz functions of delayed (fuzzy) stochastic switched financial systems, free weight matrix criteria can not be derived. However, by means of Lyapunov function method, design of switching law, novel fuzzy assumption, $L^{p}$ estimation technique and Laplace semigroup theory, the author presents the boundedness and LMI-based (globally) asymptotical input-to-state stability criteria of financial systems. Particularly, the globally asymptotical stability in the meaning of switching implies that when the time $t$ is big enough, the dynamic of any subsystem must approach its unique equilibrium point. Motivated by some methods of the related literature ([19-32, 35-39]), the author presents the following highlights in this paper:

$\checkmark$ It is the first paper to design a novel fuzzy condtion and a state-dependent switching law for the globally asymptotical input-to-state stability in the meaning of switching for fuzzy switched stochastic financial system with delayed feedback;

$\checkmark$ It is the first time to utilize the Laplace semigroup to derive the boundedness for fuzzy switched stochastic financial system with delayed feedback, which reveals the relation between ordinary differential equations model and partial differential equations model.

Throughout of this paper, for any symmetric matrices $A$ and $B$, the matrix-form inequality $A<B$ represents that 
the symmetric matrix $(B-A)$ is a positive definite matrix.

\section{System description}

The following financial system has been investigated in many existing literature([1-9]),

$$
\left\{\begin{array}{l}
\dot{x}=z+(y-a) x \\
\dot{y}=1-b y-x^{2} \\
\dot{z}=-x-c z,
\end{array}\right.
$$

where $x$ represents the interest rate, $y$ represents the investment demand, $z$ represents the price index, $a$ represents savings, $b$ represents the unit investment cost, and $c$ represents the elasticity of commodity demand. It is well known that if $c-b-a b c \leqslant 0$, the financial system (2.1) has the unique equilibrium point $P_{2}\left(0, \frac{1}{b}, 0\right)$; if $c-b-a b c>0$, the financial system (2.1) owns three equilibrium point $P_{1}\left(\theta, \frac{1+a c}{c}, \frac{-\theta}{c}\right), P_{2}\left(0, \frac{1}{b}, 0\right), P_{3}\left(-\theta, \frac{1+a c}{c}, \frac{\theta}{c}\right)$, where $\theta=\sqrt{\frac{c-b-a b c}{c}}$. Chaos appears in the financial system (2.1) if $c-b-a b c>0$ and $c+a-\frac{1}{b}<0$. For example, let $a=0.9, b=0.2, c=1.2$, then there is a chaos phenomenon in the financial system (2.1) (see, e.g. [2,3]). Throughout this paper, we assume that $c-b-a b c>0$.

For the equilibrium point $P_{1}\left(\theta, \frac{1+a c}{c}, \frac{-\theta}{c}\right)$, we may set

$$
\left\{\begin{array}{l}
X_{1}=x-\theta \\
X_{2}=y-\frac{1+a c}{c} \\
X_{3}=z+\frac{\theta}{c}
\end{array}\right.
$$

then the financial system (2.1) is translated into the following system:

$$
\left\{\begin{array}{l}
\dot{X}_{1}=\frac{1}{c} X_{1}+\theta X_{2}+X_{3}+X_{1} X_{2} \\
\dot{X}_{2}=-2 \theta X_{1}-b X_{2}-X_{1}^{2} \\
\dot{X}_{3}=-X_{1}-c X_{3}
\end{array}\right.
$$

or

$$
\dot{X}(t)=H_{1} X(t)+f(X(t))
$$

where the equilibrium point $P_{1}\left(\theta, \frac{1+a c}{c}, \frac{-\theta}{c}\right)$ of the financial system (2.2) is corresponding to the null solution of the system (2.3) or (2.4), and $A$ and $f$ are defined as follows,

$$
H_{1}=\left(\begin{array}{ccc}
\frac{1}{c} & \theta & 1 \\
-2 \theta & -b & 0 \\
-1 & 0 & -c
\end{array}\right), \quad f(X)=\left(\begin{array}{c}
X_{1} X_{2} \\
-X_{1}^{2} \\
0
\end{array}\right),
$$


Similarly, for the equilibrium point $P_{2}\left(0, \frac{1}{b}, 0\right)$, let

$$
\begin{gathered}
\left\{\begin{array}{l}
X_{1}=x \\
X_{2}=y-\frac{1}{b} \\
X_{3}=z,
\end{array}\right. \\
\left\{\begin{array}{l}
\dot{X}_{1}=\left(\frac{1}{b}-a\right) X_{1}+X_{3}+X_{1} X_{2} \\
\dot{X}_{2}=-b X_{2}-X_{1}^{2} \\
\dot{X}_{3}=-X_{1}-c X_{3},
\end{array}\right.
\end{gathered}
$$

or

$$
\dot{X}=H_{2} X+f(X)
$$

where $X=\left(X_{1}, X_{2}, X_{3}\right)^{T}$,

$$
H_{2}=\left(\begin{array}{ccc}
\frac{1}{b}-a & 0 & 1 \\
0 & -b & 0 \\
-1 & 0 & -c
\end{array}\right)
$$

For the equilibrium point $P_{3}\left(-\theta, \frac{1+a c}{c}, \frac{\theta}{c}\right)$, we may set

$$
\begin{gathered}
\left\{\begin{array}{l}
X_{1}=x+\theta \\
X_{2}=y-\frac{1+a c}{c} \\
X_{3}=z-\frac{\theta}{c}
\end{array}\right. \\
\left\{\begin{array}{l}
\dot{X}_{1}=\frac{1}{c} X_{1}-\theta X_{2}+X_{3}+X_{1} X_{2} \\
\dot{X}_{2}=2 \theta X_{1}-b X_{2}-X_{1}^{2} \\
\dot{X}_{3}=-X_{1}-c X_{3},
\end{array}\right.
\end{gathered}
$$

or

$$
\dot{X}(t)=H_{3} X(t)+f(X(t))
$$

where

$$
H_{3}=\left(\begin{array}{ccc}
\frac{1}{c} & -\theta & 1 \\
2 \theta & -b & 0 \\
-1 & 0 & -c
\end{array}\right)
$$


In the real financial market, the economic system is a dynamic market with randomness. Economic activities do not necessarily pursue a fixed interest rate. The null solution of the following switched system

$$
\dot{X}(t)=H_{\sigma} X(t)+f(X(t)), \quad \sigma \in \mathfrak{T}, \text { where } \mathfrak{T}=\{1\}, \text { or }\{1,2\}, \text { or }\{1,2,3\}
$$

corresponds to a series of equilibrium points $P_{\sigma}$ with respective interest rates. The switched system (2.14) means that the financial system is adjusted macroscopically, or the market adjusts itself when the economic situation changes to a critical value. For example, when the economic crisis breaks out, in order to promote economic development, the interest rate is adjusted to zero or even negative. Thereby, the global asymptotic stability of the switched system (2.14) reflects the activity of real financial market and the stability.

In financial management, the Uncertainty of parameters sampling conforms to the fuzzy model to a great extent. So we consider the following T-S fuzzy rule for the financial system (2.14).

Fuzzy Rule $r$ : IF $\hat{\omega}_{1}(t)$ is $v_{r 1}$ and $\cdots$ and $\hat{\omega}_{K}(t)$ is $v_{r k}$ THEN

$$
\dot{X}(t)=H_{\sigma r} X(t)+f(X(t))
$$

where $\hat{\omega}_{k}(t)(k=1,2, \cdots, \kappa)$ is the premise variable, $v_{r k}(r=1,2, \cdots, n ; k=1,2, \cdots, \kappa)$ is the fuzzy set that is characterized by membership function. And $n$ is the number of the IF-THEN rules, $\kappa$ is the number of the premise variables.

In view of a standard fuzzy inference method, (2.15) can be inferred as follows,

$$
\dot{X}(t)=\sum_{r=1}^{n} \varrho_{r}(\hat{\omega}(t)) H_{\sigma r} X(t)+f(X(t)),
$$

where $\hat{\omega}(t)=\left[\hat{\omega}_{1}(t), \hat{\omega}_{2}(t), \cdots, \hat{\omega}_{\kappa}(t)\right], \varrho_{r}(\hat{\omega}(t))=\frac{\bar{w}_{r}(\hat{\omega}(t))}{\sum_{k=1}^{n} \bar{w}_{k}(\hat{\omega}(t))}, \bar{w}_{r}(\hat{\omega}(t))=\prod_{j=1}^{\kappa} v_{r j}(\hat{\omega}(t)): R^{\kappa} \rightarrow[0,1](r=1,2, \cdots, n)$ denotes the grade of membership of $\hat{\omega}(t)$ in $v_{r j}$ with respect to the fuzzy rule $r$. $\varrho_{r}$ can be regarded as the normalized weight of each IF-THEN rule, satisfying

$$
\varrho_{r}(\hat{\omega}(t)) \geqslant 0, \quad \sum_{r=1}^{n} \varrho_{r}(\hat{\omega}(t))=1 .
$$

Remark 1. Since the important economic parameters, such as savings, the unit investment cost, and the elasticity of commodity demand, usually come from some small sample statistics, we hope that the financial system can be stabilized in a certain range of parameter ambiguity.

Since some stochastic disturbance factors in the real financial market, such as the input and outflow of foreign capital, these additional funds will produce immediate and delayed stochastic disturbance to some important financial parameters, such as savings, the unit investment cost, and the elasticity of commodity demand. So, in this paper, we have to consider the following delayed feedback stochastic financial system:

$$
\left\{\begin{array}{l}
d X(t)=\left[\sum_{r=1}^{n} \varrho_{r}(\hat{\omega}(t)) H_{\sigma r} X(t)+f(X(t))+v(t)\right] d t+\varphi_{\sigma}(X(t), X(t-\tau(t))) d w(t), \quad t \geqslant 0 \\
X(s)=\xi(s), \quad s \in[-\tau, 0]
\end{array}\right.
$$


where $v$ is the external input, time delay $\tau(t)$ satisfies $\tau(t) \in[0, \tau]$, and $X(t-\tau(t))$ is the delayed feedback state variable. The noise perturbation $\varphi: R^{3} \times R^{3} \rightarrow R^{3 \times 3}$ is a Borel measurable function with $\varphi_{\sigma}(X(t), X(t-\tau(t)))=\left(\varphi_{1}, \varphi_{2}, \varphi_{3}\right)^{T}$ and $\varphi_{i}=\left(\varphi_{\sigma i 1}\left(X_{i}(t), X_{i}(t-\tau(t))\right), \varphi_{\sigma i 2}\left(X_{i}(t), X_{i}(t-\tau(t))\right), \varphi_{\sigma i 3}\left(X_{i}(t), X_{i}(t-\tau(t))\right)\right)$, and $w(t)=\left(w_{1}(t), w_{2}(t), w_{3}(t)\right)^{T} \in$ $R^{3}$ is a 3-dimensional Brownian motion defined on a complete probability space $(\Upsilon, \mathcal{F}, \mathbb{P})$ with a natural filtration $\left\{F_{t}\right\}_{t \geqslant 0}$ generated by $\{w(s): 0 \leqslant s \leqslant t\}$, where we associate $\Upsilon$ with the canonical space generated by $w(t)$, and denoted by $\mathcal{F}$ the associated $\sigma$-algebra generated by $w(t)$ with the probability measure $\mathbb{P}$, and $\mathbb{E}\{d w(t)\}=0$. Besides, $\mathbb{E}\left\{d w_{i}(t) d w_{j}(t)\right\}=d t$ if $i=j$, and $\mathbb{E}\left\{d w_{i}(t) d w_{j}(t)\right\}=0$ if $i \neq j$, where $i, j=1,2,3$.

If the fuzzy factors are ignored, the system (2.17) is degenerated into the following system:

$$
\left\{\begin{array}{l}
d X(t)=\left[H_{\sigma} X(t)+f(X(t))+v(t)\right] d t+\varphi_{\sigma}(X(t), X(t-\tau(t))) d w(t), \quad t \geqslant 0 \\
X(s)=\xi(s), \quad s \in[-\tau, 0]
\end{array}\right.
$$

Definition 1 ([32, Definition 3]). The switched system (2.16) is said to be globally asymptotically stable in the meaning of switching if the zero solution of the switched system (2.18) is be globally asymptotically stable. Particularly, in the case of $\mathfrak{T}=\{1\}$, the switched system (2.16) is globally exponentially stable (in the classical meaning) if the zero solution of the switched system (2.16) is be globally asymptotically stable.

Remark 2. The globally asymptotical stability in the meaning of switching means that when the time $t>0$ is big enough, and the system is switched into any ith subsystem, the dynamic of the subsystem must approach its equilibrium point $P_{i}$. For example, the financial crisis breaks out, the financial system is switched into a subsystem, say, the 2 th subsystem whose equilibrium point is $P_{2}$, and the dynamic of the subsystem must approach $P_{2}$ if the time $t>0$ is big enough. Moreover, if the stability is local, then the dynamic of the subsystem may not approach $P_{2}$ due to various different initial values. Besides, the global financial crisis often erupts periodically, which illuminates that the global stability in the classical sense is actually meaningless. So the stability in the meaning of switching proposed in this paper is suitable and appropriate.

In this paper, we assume

(A1) There are constant symmetric matrices $\Lambda_{\sigma 1}$ and $\Lambda_{\sigma 2}$ with $\Lambda_{\sigma 2}<\alpha_{\sigma} I$ such that

$$
\operatorname{trace}\left[\varphi_{\sigma}^{T}(u, v) \varphi_{\sigma}(u, v)\right] \leqslant u^{T} \Lambda_{\sigma 1} u+v^{T} \Lambda_{\sigma 2} v
$$

where $I$ represents the identity matrix.

The following estimate condition for fuzzy parameters is considered for the fuzzy system (2.17):

(A2) For any given mode $\sigma$, there exists correspondingly a constant matrix $E_{\sigma}$ such that

$$
\sum_{r=1}^{n} \varrho_{r}(\hat{\omega}(t))\left(H_{\sigma r}+H_{\sigma r}^{T}\right)<\frac{1}{n} \sum_{r=1}^{n}\left(H_{\sigma r}+H_{\sigma r}^{T}\right)+E_{\sigma}+E_{\sigma}^{T}
$$

Remark 3. The fuzzy estimate condition is feasible, which can be verified in (5.3)-(5.5) of Example 5.2 below. 
Lemma 1.1([28,29]). Let $\Omega \subset \mathbb{R}^{N}(N \in \mathbb{N})$ be a bounded domain with smooth boundary and let $\Delta$ denote the Laplacian in $L^{s}(\Omega)$ with domain

$$
\left\{z \in W^{2, s}(\Omega) \mid \nabla \cdot v=0 \text { on } \partial \Omega\right\}
$$

for $s \in(1, \infty)$. Then the operator $-\Delta+1$ is sectorial and possesses closed fractional powers $(-\Delta+1)^{\delta}, \delta \in(0,1)$, with dense domain $D\left((-\Delta+1)^{\delta}\right)$. Moreover, the following three properties hold.

(i) If $m \in\{0,1\}, p \in[1, \infty]$ and $q \in(1, \infty)$, then there exists a constant $C_{1}>0$ such that for all $z \in D\left((-\Delta+1)^{\delta}\right)$,

$$
\|z\|_{W^{m, p}(\Omega)} \leqslant C_{1}\left\|(-\Delta+1)^{\delta} z\right\|_{L^{q}(\Omega)},
$$

(ii) Suppose $p \in[1, \infty)$. Then the associated heat semigroup $\left(e^{t \Delta}\right)_{t \geqslant 0}$ maps $L^{p}(\Omega)$ into $D\left((-\Delta+1)^{\delta}\right)$ in $L^{p}(\Omega)$, and there exist constants $C_{2}>0$ and $\lambda_{2}>0$ such that

$$
\left\|(-\Delta+1)^{\delta} e^{t(\Delta-1)} z\right\|_{L^{p}(\Omega)} \leqslant C_{2} t^{-\delta} e^{-\lambda_{2} t}\|z\|_{L^{p}(\Omega)}
$$

for all $z \in L^{p}(\Omega)$ and all $t>0$.

\section{Main result in the case of non-fuzzy factors}

Define the switching law for the switched system (2.18) as follows,

switching law $\mathfrak{F}_{a}$ : At each switching we determine the next mode according to the following minimum law :

$$
\sigma(t)=\arg \min \sum_{r=1}^{n} \xi^{T}\left(\begin{array}{cc}
Q+P H_{\sigma}+H_{\sigma}^{T} P+\left(\varepsilon P+\varepsilon^{-1} \alpha_{1}^{2} P\right)+\rho \Lambda_{\sigma 1}+P \bar{D}_{\sigma}+\bar{D}_{\sigma}^{T} P+\Psi & 0 \\
0 & \rho \Lambda_{\sigma 2}-(1-\alpha) Q
\end{array}\right) \xi
$$

$\left(\mathfrak{F}_{a 1}\right)$ Choose the initial mode $\sigma(t)=i_{0}$, if $\xi\left(t_{0}\right) \in \Upsilon_{i_{0}}$.

$\left(\mathfrak{F}_{a 2}\right)$ For each $t>t_{0}$, if $\sigma\left(t^{-}\right)=i$ and $\xi \in \Upsilon_{i}$, keep $\sigma(t)=i$. On the other hand, if $\sigma\left(t^{-}\right)=i$ but $\xi \notin \Upsilon_{i}$. i.e., hitting a switching surface, choose the next mode by applying (3.1) and begin to switch.

Here, $\Upsilon_{\sigma}$ is defined as follows,

$$
\Upsilon_{\sigma}=\left\{\xi \in R^{6} \mid \xi^{T}\left(\begin{array}{cc}
Q+P H_{\sigma}+H_{\sigma}^{T} P+\left(\varepsilon P+\varepsilon^{-1} \alpha_{1}^{2} P\right)+\rho \Lambda_{\sigma 1}+P \bar{D}_{\sigma}+\bar{D}_{\sigma}^{T} P+\Psi & 0 \\
0 & \rho \Lambda_{\sigma 2}-(1-\alpha) Q
\end{array}\right) \xi<0\right\}
$$

where $\varepsilon>0, \rho>0$ are two given positive numbers, $P=\operatorname{diag}\left(p_{1}, p_{2}, p_{3}\right)$ is a given positive definite diagonal matrix with $p_{1}=p_{2}$, and $Q>0, \Psi>0$ are given symmetric matrices, respectively.

(A3) Since $X_{1}$ represents the interest rate which is actually bounded in real world, there exists the positive number $\alpha_{1}$ such that $\left|X_{1}\right| \leqslant \alpha_{1}$.

(A4) There are constant matrices $\underline{D}_{\sigma}$ and $\bar{D}_{\sigma}$ such that the external input satisfies $v(t)=D_{\sigma}(t) X(t)$ with $\underline{D}_{\sigma} \leqslant$ $D_{\sigma}(t) \leqslant \bar{D}_{\sigma}$ 
Theorem 3.1. Suppose the conditions (A1),(A3)and (A4) hold. Besides, $\dot{\tau}(t) \leqslant \alpha$, and there is a positive definite diagonal matrix $P \leqslant \rho I$ with $P=\operatorname{diag}\left(p_{1}, p_{2}, p_{3}\right)$ with $p_{1}=p_{2}$, a positive definite matrix $Q$, and a sequence of non-negative numbers $\beta_{\sigma}(\sigma \in \mathfrak{T})$ with $\sum_{\sigma \in \mathfrak{T}} \beta_{\sigma}=1$ such that

$$
\left(\begin{array}{cc}
\sum_{\sigma \in \mathfrak{T}} \beta_{\sigma}\left(P H_{\sigma}+H_{\sigma}^{T} P+\rho \Lambda_{\sigma 1}+P \bar{D}_{\sigma}+\bar{D}_{\sigma}^{T} P\right)+\left(\varepsilon P+\varepsilon^{-1} \alpha_{1}^{2} P\right)+Q+\Psi & 0 \\
0 & \rho \sum_{\sigma \in \mathfrak{T}} \Lambda_{\sigma 2}-(1-\alpha) Q
\end{array}\right)<0
$$

then the zero solution of the system (2.18) is the global asymptotical input-to-state stability. That is, the switched system (2.18) is said to be the global asymptotical input-to-state stability in the meaning of switching.

Proof. Consider the following Lyapunov-Krasovskii function

$$
V(t, X)=X^{T}(t) P X(t)+\int_{t-\tau(t)}^{t} X^{T}(s) Q X(s) d s .
$$

The condition $(\mathrm{A} 3)$ and $P=\operatorname{diag}\left(p_{1}, p_{1}, p_{3}\right)$ yield

$$
\begin{aligned}
& f(X)=\left(\begin{array}{c}
f_{1}(X) \\
f_{2}(X) \\
f_{3}(X)
\end{array}\right)=\left(\begin{array}{c}
X_{1} X_{2} \\
-X_{1}^{2} \\
0
\end{array}\right), \quad X^{T} P f(X)+[\sqrt{P} f(X)]^{T}(\sqrt{P} X) \leqslant \varepsilon X^{T} P X+\varepsilon^{-1} f^{T}(X) P f(X), P=\operatorname{diag}\left(p_{1}, p_{2}, p_{3}\right), p_{1}=p_{2} \\
\Rightarrow & X^{T} P f(X)+f^{T}(X) P X \leqslant \varepsilon X^{T} P X+\varepsilon^{-1}\left[X_{1}^{2}\left(p_{2} X_{1}^{2}+p_{1} X_{2}^{2}\right)\right] \leqslant X^{T}\left(\varepsilon P+\varepsilon^{-1} \alpha_{1}^{2} P\right) X .
\end{aligned}
$$

Let $R_{+}$be the set of positive numbers. Denote by $C^{1,2}\left(R_{+} \times R^{n} \rightarrow R_{+}\right)$the family of nonnegative functions $V(t, X)$ on $R_{+} \times R^{n}$ which are continuous once differentiable in $t$ and twice differentiable in $X$. For each such $V$, one can define an operator $\mathcal{L} V$ associated with equations (2.18) as

$$
\begin{aligned}
\mathcal{L} V= & V_{t}(t, X(t))+V_{X}(t, X(t))\left[H_{\sigma} X(t)+f(X(t))+v(t)\right] \\
& +\frac{1}{2} \operatorname{trace}\left[\varphi_{\sigma}^{T}(X(t), X(t-\tau(t))) V_{X X}(t, X(t)) \varphi_{\sigma}(X(t), X(t-\tau(t)))\right] \\
\leqslant & X^{T}(t) Q X(t)-(1-\dot{\tau}(t)) X^{T}(t-\tau(t)) Q X(t-\tau(t))+2 X^{T}(t) P\left[H_{\sigma} X(t)+f(X(t))+D_{\sigma}(t) X(t)\right] \\
& +\rho \operatorname{trace}\left[\varphi_{\sigma}^{T}(X(t), X(t-\tau(t))) \varphi_{\sigma}(X(t), X(t-\tau(t)))\right] \\
\leqslant & X^{T}(t)\left[Q+P H_{\sigma}+H_{\sigma}^{T} P+\left(\varepsilon P+\varepsilon^{-1} \alpha_{1}^{2} P\right)+\rho \Lambda_{\sigma 1}+P \bar{D}_{\sigma}+\bar{D}_{\sigma}^{T} P\right] X(t)+X^{T}(t-\tau(t))\left(\rho \Lambda_{\sigma 2}-(1-\alpha) Q\right) X(t-\tau(t)) .
\end{aligned}
$$

where

$$
V_{X}(t, X(t))=\left(\frac{\partial V(t, X(t))}{\partial X_{1}}, \frac{\partial V(t, X(t))}{\partial X_{2}}, \frac{\partial V(t, X(t))}{\partial X_{3}}\right), \quad V_{X X}(t, X(t))=\left(\frac{\partial^{2} V(t, X(t))}{\partial X_{i} \partial X_{j}}\right)_{3 \times 3} .
$$

Set $\xi=\left(X^{T}(t), X^{T}(t-\tau(t))\right)^{T}$, one can claim 


$$
\begin{aligned}
\mathcal{L} V & \leqslant \xi^{T}\left(\begin{array}{cc}
Q+P H_{\sigma}+H_{\sigma}^{T} P+\left(\varepsilon P+\varepsilon^{-1} \alpha_{1}^{2} P\right)+\rho \Lambda_{\sigma 1}+P \bar{D}_{\sigma}+\bar{D}_{\sigma}^{T} P & 0 \\
0 & \rho \Lambda_{\sigma 2}-(1-\alpha) Q
\end{array}\right) \xi \\
& <\xi^{T}\left(\begin{array}{cc}
-\Psi & 0 \\
0 & 0
\end{array}\right) \xi<0 .
\end{aligned}
$$

In fact, it follows by (3.2) and the proof by contradiction that $\bigcup_{\sigma \in \mathfrak{T}} \Upsilon_{\sigma}=R^{6} \backslash\{0\}$. On one hand, when $\sigma\left(t^{-}\right)=i$ and $\xi(t) \in \Upsilon_{i}$, then keep $\sigma(t)=i$, and (3.5) holds. On the other hand, when $\sigma\left(t^{-}\right)=i$ and $\xi(t) \notin \Upsilon_{i}$, which means that the trajectory hits a switching surface, and is chosen by the minimum law (3.2). At the same time, $\xi \in \Upsilon_{j}(j \neq i)$, and then (3.5) holds.

Besides,

$$
\begin{aligned}
d V(t) & =\mathcal{L} V(t) d t+V_{X}(t) \varphi_{\sigma}(X(t), X(t-\tau(t))) d w(t) \\
& \leqslant \xi^{T}(t) \Upsilon_{\sigma} \xi(t)+2 X^{T}(t) P \varphi_{\sigma}(X(t), X(t-\tau(t))) d w(t)
\end{aligned}
$$

So it follows by (3.6) that

$$
\begin{aligned}
\mathbb{E} V(t+\varepsilon)-\mathbb{E} V(t) & =\int_{t}^{t+\varepsilon} \mathbb{E} \mathcal{L} V(s) d s+\mathbb{E} \int_{t}^{t+\varepsilon} 2 X^{T}(s) P \varphi_{\sigma}(X(s), X(s-\tau(s))) d w(s) \\
& =\int_{t}^{t+\varepsilon} \mathbb{E} \mathcal{L} V(s) d s
\end{aligned}
$$

which implies that

$$
D^{+} \mathbb{E} V(t)<0
$$

Moreover, it is obvious that

$$
\left(\lambda_{\min } P\right)|X(t)|^{2} \leqslant X^{T}(t) P X(t) \leqslant V(t) \leqslant\left(\lambda_{\max } P+\tau \lambda_{\max } Q\right)\left\|X_{t}\right\|_{\tau},
$$

where

$$
\left\|X_{t}\right\|_{\tau}=\sup _{s \in[-\tau, 0]}|X(t+s)|
$$

Combining (3.8) and (3.9) results in that the zero solution of the system (2.18) is the global asymptotical input-tostate stability. That is, the switched system (2.18) is said to be the global asymptotical input-to-state stability in the meaning of switching.

Remark 4. The global stability in the meaning of switching has practical significance. In fact, the global financial crisis often erupts periodically, which illuminates that the global stability in the classical sense is actually meaningless.

\section{Main result in the case of fuzzy system}

Define the switching law as follows,

switching law $\mathfrak{F}$ : At each switching we determine the next mode according to the following minimum law : 


$$
\sigma(t)=\arg \min \sum_{r=1}^{n} X^{T}\left[\frac{1}{n} \sum_{r=1}^{n}\left(H_{\sigma r}+H_{\sigma r}^{T}\right)+E_{\sigma}+E_{\sigma}^{T}+\Lambda_{\sigma 1}+e^{\varsigma \tau} q \alpha_{\sigma} I+\bar{D}_{\sigma}+\bar{D}_{\sigma}^{T}+\Psi\right] X .
$$

$\left(\mathfrak{F}_{1}\right)$ Choose the initial mode $\sigma(t)=i_{0}$, if $X\left(t_{0}\right) \in \Upsilon_{i_{0}}$.

$\left(\mathfrak{F}_{2}\right)$ For each $t>t_{0}$, if $\sigma\left(t^{-}\right)=i$ and $X \in \Upsilon_{i}$, keep $\sigma(t)=i$. On the other hand, if $\sigma\left(t^{-}\right)=i$ but $X \notin \Upsilon_{i}$. i.e., hitting a switching surface, choose the next mode by applying (4.1) and begin to switch.

Here, $\Psi$ is a positive definite symmetric matrix with $\lambda_{\min } \Psi=\lambda>0$, and $\Upsilon_{\sigma}$ is defined as follows,

$$
\Upsilon_{\sigma}=\left\{X \in R^{3} \mid X^{T}\left(\frac{1}{n} \sum_{r=1}^{n}\left(H_{\sigma r}+H_{\sigma r}^{T}\right)+E_{\sigma}+E_{\sigma}^{T}+\Lambda_{\sigma 1}+e^{\varsigma \tau} q \alpha_{\sigma} I+\bar{D}_{\sigma}+\bar{D}_{\sigma}^{T}+\Psi\right) X<0\right\},
$$

where $\lambda_{\min } \Psi$ represents the minimum of all the eigenvalues of the symmetric matrix $\Psi>0$.

Theorem 4.1. Suppose that the conditions (A1),(A2) and (A4) hold, and there is a sequence of nonnegative constants $\beta_{\sigma}(\sigma=1,2,3)$ with $\sum_{\sigma=1}^{3} \beta_{\sigma}=1$ and $0 \leqslant \beta_{\sigma} \leqslant 1$ and positive constants $\varsigma>0$ and $q>1$ such that

$$
\sum_{\sigma=1}^{3} \beta_{\sigma}\left[\frac{1}{n} \sum_{r=1}^{n}\left(H_{\sigma r}+H_{\sigma r}^{T}\right)+E_{\sigma}+E_{\sigma}^{T}+\Lambda_{\sigma 1}+e^{\varsigma \tau} q \alpha_{\sigma} I+\bar{D}_{\sigma}+\bar{D}_{\sigma}^{T}\right]+\Psi<0
$$

then the fuzzy switched system (2.17) is exponential input-to-state stability.

Proof. Consider the following Lyapunov function:

$$
V(t)=V(t, X(t))=X^{T}(t) X(t)
$$

Let $R_{+}$be the set of positive numbers. Denote by $C^{1,2}\left(R_{+} \times R^{n} \rightarrow R_{+}\right)$the family of nonnegative functions $V(t, X)$ on $R_{+} \times R^{n}$ which are continuous once differentiable in $t$ and twice differentiable in $X$. For each such $V$, we define an operator $\mathcal{L} V$ associated with equations (2.17) as

$$
\begin{aligned}
\mathcal{L} V= & V_{t}(t, X(t))+V_{X}(t, X(t))\left[\left(\sum_{r=1}^{n} \varrho_{r}(\hat{\omega}(t)) H_{\sigma r}\right) X(t)+f(X(t))+v(t)\right] \\
& +\frac{1}{2} \operatorname{trace}\left[\varphi_{\sigma}^{T}(X(t), X(t-\tau(t))) V_{X X}(t, X(t)) \varphi_{\sigma}(X(t), X(t-\tau(t)))\right] \\
\leqslant & X^{T}(t)\left(\frac{1}{n} \sum_{r=1}^{n}\left(H_{\sigma r}+H_{\sigma r}^{T}\right)+E_{\sigma}+E_{\sigma}^{T}+\bar{D}_{\sigma}+\bar{D}_{\sigma}^{T}\right) X(t) \\
& +\left(X^{T}(t) \Lambda_{\sigma 1} X(t)+X^{T}(t-\tau(t)) \Lambda_{\sigma 2} X(t-\tau(t))\right)
\end{aligned}
$$

where

$$
V_{X}(t, X(t))=\left(\frac{\partial V(t, X(t))}{\partial X_{1}}, \frac{\partial V(t, X(t))}{\partial X_{2}}, \frac{\partial V(t, X(t))}{\partial X_{3}}\right), \quad V_{X X}(t, X(t))=\left(\frac{\partial^{2} V(t, X(t))}{\partial X_{i} \partial X_{j}}\right)_{3 \times 3}
$$

Let $\gamma$ be a positive number with $0<\gamma<\min \left\{\lambda, \varsigma, \frac{1}{2} \lambda_{2}\right\}$ and

$$
U(t, X(t))= \begin{cases}e^{\gamma t} X^{T}(t) X(t), & t \geqslant 0 \\ X^{T}(t) X(t), & t \in[-\tau, 0]\end{cases}
$$


where $\lambda_{2}>0$ is the first positive eigenvalue of the following Neumann boundary problem

$$
\left\{\begin{aligned}
(-\Delta+1) z=\lambda z, & \text { in } \Omega, \\
\partial_{v} z=0, & \text { on } \partial \Omega,
\end{aligned}\right.
$$

and $\Omega$ is a bounded domain in $R^{3}$ with smooth boundary $\partial \Omega$.

It is obvious that $U$ is continuous for $t \geqslant-\tau$. For $t \geqslant 0$ and $\gamma>0$,

$$
d U(t, X(t))=e^{\gamma t}\left(\gamma X^{T}(t) X(t) d t+d\left[X^{T}(t) X(t)\right]\right)
$$

Ito formula yields

$$
d V(t)=\mathcal{L} V(t) d t+V_{X}(t) \varphi_{\sigma}(X(t), X(t-\tau(t))) d w(t)
$$

and hence

$$
d U(t, X(t))=e^{\gamma t}\left(\gamma X^{T}(t) X(t) d t+\mathcal{L} V(t) d t+V_{X}(t) \varphi_{\sigma}(X(t), X(t-\tau(t))) d w(t)\right)
$$

Integrating the above inequality from $t$ to $t+\varepsilon$, and taking the mathematical expectation, one may derive that, for all $t \geqslant 0$ and any $\varepsilon>0$,

$$
\mathbb{E} U(t+\varepsilon, X(t+\varepsilon))-\mathbb{E} U(t, X(t))=\mathbb{E} \int_{\varepsilon}^{t+\varepsilon} e^{\gamma s}\left(\gamma X^{T}(s) X(s)+\mathcal{L} V(s)\right) d s
$$

and then

$$
D^{+} \mathbb{E} U(t, X(t))=e^{\gamma t}\left(\gamma \mathbb{E}\left[X^{T}(t) X(t)\right]+\mathbb{E} \mathcal{L} V(t)\right)
$$

Now we claim that there is a positive constants $C_{0}>1$ and $K \in R$ with $K>1$ such that

$$
\mathbb{E} U(t, X(t)) \leqslant K C_{0} \mathbb{E}\|\xi\|_{\tau}^{2}, \quad \forall t \geqslant 0
$$

where $\|\xi\|_{\tau}=\sup _{s \in[-\tau, 0]} \sqrt{\xi^{T}(s) \xi(s)}$

Indeed, suppose this assertion is not true, then it is not difficult to prove (see, e.g. [7]) that there exists positive constant $q$ with $K \geqslant q>1$ such that

$$
\mathbb{E} U(t, X(t)) \leqslant K C_{0} \mathbb{E}\|\xi\|_{\tau}^{2}, \quad t \in\left[-\tau, t^{*}\right]
$$

and

$$
\mathbb{E} U\left(t^{* *}, X\left(t^{* *}\right)\right)=\frac{1}{q} K C_{0} \mathbb{E}\|\xi\|_{\tau}^{2} ; \mathbb{E} U\left(t^{* *}, X\left(t^{* *}\right)\right) \leqslant \mathbb{E} U(t, X(t)) \leqslant \mathbb{E} U\left(t^{*}, X\left(t^{*}\right)\right)=K C_{0} \mathbb{E}\|\xi\|_{\tau}^{2}, \quad \forall t \in\left[t^{* *}, t^{*}\right] .
$$

It follows from (4.6), (4.7) and the definition of $U(t, X(t))$ that for $s \in[-\tau, 0]$ and $t \in\left[t^{* *}, t^{*}\right]$ 


$$
\begin{aligned}
e^{\varsigma s} \mathbb{E}\left[X^{T}(t+s) X(t+s)\right] & \leqslant e^{\gamma s} \mathbb{E}\left[X^{T}(t+s) X(t+s)\right] \\
& =\left\{\begin{array}{cc}
e^{-\gamma t} \mathbb{E} U(t+s, X(t+s)), & t+s \geqslant 0 \\
e^{\gamma s} \mathbb{E} U(t+s, X(t+s)), & t+s \leqslant 0
\end{array}\right. \\
& \leqslant e^{-\gamma t} \mathbb{E} U(t+s, X(t+s)) \\
& \leqslant e^{-\gamma t} q e^{\gamma t} \mathbb{E}\left[X^{T}(t) X(t)\right] \\
& =q \mathbb{E}\left[X^{T}(t) X(t)\right],
\end{aligned}
$$

which yields that for any $s \in[-\tau, 0]$,

$$
\mathbb{E}\left[X^{T}(t-\tau(t)) X(t-\tau(t))\right] \leqslant e^{\varsigma \tau} q \mathbb{E} X^{T}(t) X(t) .
$$

Combining (4.9) and (A1) results in

$$
\mathbb{E}\left[X^{T}(t-\tau(t)) \Lambda_{\sigma 2} X(t-\tau(t))\right] \leqslant e^{\varsigma \tau} q \alpha_{\sigma} \mathbb{E}\left[X^{T}(t) X(t)\right]
$$

which derives

$$
\begin{aligned}
\mathbb{E} \mathcal{L} V \leqslant & \mathbb{E}\left[X^{T}(t)\left(\frac{1}{n} \sum_{r=1}^{n}\left(H_{\sigma r}+H_{\sigma r}^{T}\right)+E_{\sigma}+E_{\sigma}^{T}+\bar{D}_{\sigma}+\bar{D}_{\sigma}^{T}\right) X(t)\right] \\
& +\mathbb{E}\left(X^{T}(t) \Lambda_{\sigma 1} X(t)+X^{T}(t-\tau(t)) \Lambda_{\sigma 2} X(t-\tau(t))\right) \\
\leqslant & \mathbb{E}\left[X^{T}(t)\left(\frac{1}{n} \sum_{r=1}^{n}\left(H_{\sigma r}+H_{\sigma r}^{T}\right)+E_{\sigma}+E_{\sigma}^{T}+\Lambda_{\sigma 1}+e^{\varsigma \tau} q \alpha_{\sigma} I+\bar{D}_{\sigma}+\bar{D}_{\sigma}^{T}\right) X(t)\right]
\end{aligned}
$$

For any given $t \geqslant t_{0}$, according to the switching law $\mathfrak{F}$, when $\sigma\left(t^{-}\right)=i$ and $X(t) \in \Upsilon_{i}$, then keep $\sigma(t)=i$, and we can conclude that

$$
\begin{aligned}
\mathbb{E} \mathcal{L} V(t, X(t)) & \leqslant \mathbb{E}\left[X^{T}(t)\left(\frac{1}{n} \sum_{r=1}^{n}\left(H_{\sigma r}+H_{\sigma r}^{T}\right)+E_{\sigma}+E_{\sigma}^{T}+\Lambda_{\sigma 1}+e^{\varsigma \tau} q \alpha_{\sigma} I+\bar{D}_{\sigma}+\bar{D}_{\sigma}^{T}\right) X(t)\right] \\
& \leqslant \mathbb{E}\left[X^{T}(t)(-\Psi) X(t)\right] \leqslant-\lambda \mathbb{E}\left[X^{T} X\right]=-\lambda \mathbb{E} V(t, X(t)) .
\end{aligned}
$$

When $\sigma\left(t^{-}\right)=i$ and $X(t) \notin \Upsilon_{i}$, which means that the trajectory hits a switching surface. On the other hand, it is not difficult to deduce from (4.2) that $\bigcup_{i=1}^{3} \Upsilon_{i}=R^{3} \backslash\{0\}$, which together with the minimum law (4.1) yields (4.12), too.

(4.12) together with (4.4) implies

$$
\begin{aligned}
D^{+} \mathbb{E} U(t, X(t)) & =e^{\gamma t}\left(\gamma \mathbb{E}\left[X^{T}(t) X(t)\right]+\mathbb{E} \mathcal{L} V(t)\right) \\
& \leqslant e^{\gamma t}\left((\gamma-\lambda) \mathbb{E}\left[X^{T}(t) X(t)\right]\right)=(\gamma-\lambda) \mathbb{E} U(t, X(t)) \leqslant 0,
\end{aligned}
$$

which derives that $\mathbb{E} U\left(t^{*}, X\left(t^{*}\right)\right) \leqslant \mathbb{E} U\left(t^{* *}, X\left(t^{* *}\right)\right)$. However, (4.7) yields

$$
\mathbb{E} U\left(t^{* *}, X\left(t^{* *}\right)\right)=\frac{1}{q} K C_{0} \mathbb{E}\|\xi\|_{\tau}^{2}<K C_{0} \mathbb{E}\|\xi\|_{\tau}^{2}=\mathbb{E} U\left(t^{*}, X\left(t^{*}\right)\right) .
$$

So we have prove (4.5), which means 


$$
e^{\gamma t} X^{T}(t) X(t) \leqslant K C_{0} \mathbb{E}\|\xi\|_{\tau}^{2}, \quad \forall t \geqslant 0,
$$

or

$$
X^{T}(t) X(t) \leqslant K C_{0} \mathbb{E}\|\xi\|_{\tau}^{2} e^{-\gamma t}, \quad \forall t \geqslant 0,
$$

which implies that the fuzzy switched system (2.17) is exponential input-to-state stability.

Remark 5. It is the first paper to design and investigate the stability of switched fuzzy delayed feedback financial system with stochastic perturbation, which is in line with the fact that a prosperous and stable financial market may not necessarily pursue a equilibrium point with a fixed interest rate. In most cases, financial market is more stable through switching law under macro control and market self-regulation.

Remark 6. Although our ordinary differential equations model successfully explains the financial market stability criterion under switching law, state variables are still related to regions, such as certain countries and regions. So we consider the partial differential equations model for the financial system (2.17).

$$
\left\{\begin{aligned}
d u(t, X)= & \Delta u(t, X) d t+\left[\sum_{r=1}^{n} \varrho_{r}(\hat{\omega}(t)) H_{\sigma r} u(t, X)+f(u(t, X))-v(t, X)\right] d t \\
& +\varphi_{\sigma}(u(t, X), u(t-\tau(t), X)) d w(t), \quad t \geqslant 0, X \in \Omega \\
u(s, X)= & \xi(s, X), \quad s \in[-\tau, 0], X \in \Omega \\
\frac{\partial u_{i}}{\partial X_{j}}= & 0, \quad X_{j} \in \partial \Omega, i, j=1,2,3,
\end{aligned}\right.
$$

where $\Delta$ is the Laplace operator(see, e.g. [28, 30,31]).

Remark 7. Considering that the interest rate, the investment demand and the price index are usually invariable in a country, we see, the state variable $u(t, X)=\left(u_{1}(t, X), u_{2}(t, X), u_{3}(t, X)\right)^{T}$ is actually uniform with respect to the region, which implies that the system (4.14) is exactly the system (2.17). That is, $u_{i}(t, X)$ of the system (4.14) is exactly equivalent to $X_{i}(t)$ in the system (2.17).

Remark 8. From [40, Lemma 2.3] in the case of $p=2$, we know that the fuzzy system (4.14) is exponentially stable under the same assumptions of Theorem 4.1.

To obtain the boundedness criterion of the system (4.14) or (2.17), we need the following assumption on the Laplace operator.

(A3) There exist $M>0$ and $\zeta>0$ such that $\left\|e^{t \Delta}\right\| \leqslant M e^{-\zeta t}$.

Below, we assume that the condition (A3) holds. Besides, due to the meaning of Remark 7, we propose the boundedness definition on the system (2.17) or (4.14). 
Definition 2. The fuzzy system (2.17) is said to be bounded under the meaning of $L^{\infty}$ if for any given $T>\tau_{1}>0$ such that for all $t \in\left[\tau_{1}, T\right]$, the each state variable $u_{i}(t, X)$ of the system (4.14) satisfies

$$
\left\|u_{i}(t, \cdot)\right\|_{L^{\infty}(\Omega)} \leqslant C, \quad \forall i=1,2,3
$$

Remark 9. The initial function $\xi(s)$ is bounded for $s \in[-\tau, 0]$,

Theorem 4.2. Under the assumptions of Theorem 4.1, the fuzzy system (2.17) is bounded under the meaning of $L^{\infty}$.

In order to verify the correctness of Theorem 4.2, we may firstly present the following technical lemma with regard to the system (4.14).

Lemma 4.3. Under the same assumptions of Theorem 4.1, the fuzzy system (4.14) is bounded under the meaning of $L^{\infty}$

Proof. For the convenience of the proof, we may rewrite the system (4.14) as follows,

$$
\left\{\begin{array}{l}
d u_{i}(t, X)=\Delta u_{i}(t, X) d t+\left[\sum_{r=1}^{n} \varrho_{r}(\hat{\omega}(t)) \sum_{j=1}^{3} h_{\sigma r i j} u_{j}(t, X)+f_{i}(u(t, X))+v_{i}(t, X)\right] d t \\
+\sum_{j=1}^{3} \varphi_{\sigma i j}\left(u_{i}(t, X), u_{i}(t-\tau(t), X)\right) d w_{j}(t), \quad t \geqslant 0, X \in \Omega \\
u(s, X)=\xi(s, x), \quad s \in[-\tau, 0], x \in \Omega \\
\frac{\partial u_{i}}{\partial X_{j}}=0, \quad X_{j} \in \partial \Omega, i, j=1,2,3
\end{array}\right.
$$

where $H_{\sigma r}=\left(h_{\sigma r i j}\right)_{3 \times 3}$.

It follows from the system (4.15) that for any given $\tau_{1}>0$,

$$
\left\{\begin{array}{l}
d u_{i}(t, X)=\left[\Delta u_{i}(t, X)-u_{i}(t, X)\right] d t+\left[\sum_{r=1}^{n} \varrho_{r}(\hat{\omega}(t)) \sum_{j=1}^{3} h_{\sigma r i j} u_{j}(t, X)+f_{i}(u(t, X))\right. \\
\left.+v_{i}(t, X)+u_{i}(t, X)\right] d t+\sum_{j=1}^{3} \varphi_{\sigma i j}\left(u_{i}(t, X), u_{i}(t-\tau(t), X)\right) d w_{j}(t), \quad t \geqslant 0, X \in \Omega \\
u(s, X)=\xi(s, X), \quad s \in[-\tau, 0], x \in \Omega \\
\frac{\partial u_{i}}{\partial X_{j}}=0, \quad X_{j} \in \partial \Omega, i, j=1,2,3
\end{array}\right.
$$


which implies that for any given $\tau_{1}>0$,

$$
\begin{aligned}
u_{i}(t, X)= & e^{t(\Delta-1)} u_{i}\left(\tau_{1}, X\right)+\int_{\tau_{1}}^{t} e^{(t-s)(\Delta-1)} \sum_{r=1}^{n} \varrho_{r}(\hat{\omega}(s)) \sum_{j=1}^{3} h_{\sigma r i j} u_{j}(s, X) d s \\
& +\int_{\tau_{1}}^{t} e^{(t-s)(\Delta-1)}\left[f_{i}(u(s, X))+v_{i}(t, X)+u_{i}(s, X)\right] d s \\
& +\int_{\tau_{1}}^{t} e^{(t-s)(\Delta-1)} \sum_{j=1}^{3} \varphi_{\sigma i j}\left(u_{i}(s, X), u_{i}(s-\tau(s), X)\right) d w_{j}(s) .
\end{aligned}
$$

We can see it from the proof of Theorem 4.1 that

$$
e^{\gamma t} X^{T}(t) X(t) \leqslant K C_{0} \mathbb{E}\|\xi\|_{\tau}^{2}, \quad \forall t \geqslant 0
$$

or

$$
e^{\gamma t} \sum_{i=1}^{3} X_{i}^{2} \leqslant K C_{0} \mathbb{E}\|\xi\|_{\tau}^{2}, \quad \forall t \geqslant 0
$$

which implies that

$$
e^{2 \gamma t} f_{i}^{2}\left(X_{i}\right) \leqslant e^{2 \gamma t} f^{T}(X) f(X)=e^{2 \gamma t}\left(X_{1}^{2} X_{2}^{2}+X_{1}^{4}\right) \leqslant e^{2 \gamma t} \sum_{i=1}^{3} X_{i}^{2} \sum_{i=1}^{3} X_{i}^{2} \leqslant\left(K C_{0} \mathbb{E}\|\xi\|_{\tau}^{2}\right)^{2}
$$

then we know from Remark 8 that

$$
e^{\gamma t} \sum_{i=1}^{3}\left\|u_{i}(t, X)\right\|_{L^{2}(\Omega)}^{2} \leqslant C, \text { and } e^{2 \gamma t}\left\|f_{i}^{2}\left(u_{i}(t, X)\right)\right\|_{L^{2}(\Omega)}^{2} \leqslant C^{2}, \quad t \geqslant 0,
$$

where $2 \gamma<\lambda_{2}$, and $C>0$ is a constant which is big enough.

Since $C$ is big enough, we can make a series of estimates with Lemma 1.1,

$$
\begin{aligned}
& \mathbb{E}\left\|e^{t \Delta} u_{i}\left(\tau_{1}, X\right)\right\|_{L^{\infty}(\Omega)} \\
\leqslant & C_{1} C_{2} M t^{-\delta} e^{-\lambda_{2} t} \mathbb{E}\left\|u_{i}\left(\tau_{1}, X\right)\right\|_{L^{2}(\Omega)} \leqslant C,
\end{aligned}
$$

where $\delta \in\left(\frac{1}{3}, \frac{2}{3}\right)$, and $\lambda_{2}$ is defined in (4.3).

$$
\begin{aligned}
& \mathbb{E}\left\|\int_{\tau_{1}}^{t} e^{(t-s)(\Delta-1)} \sum_{r=1}^{n} \varrho_{r}(\hat{\omega}(s)) \sum_{j=1}^{3} h_{\sigma r i j} u_{j}(s, X) d s\right\|_{L^{\infty}(\Omega)} \\
\leqslant & \sum_{r=1}^{n} \sum_{j=1}^{3}\left|h_{\sigma r i j}\right| C_{1} C_{2} M \mathbb{E} \int_{\tau_{1}}^{t}(t-s)^{-\delta} e^{-\lambda_{2}(t-s)}\left\|u_{j}(s, X)\right\|_{L^{2}(\Omega)} d s \\
\leqslant & \sum_{r=1}^{n} \sum_{j=1}^{3}\left|h_{\sigma r i j}\right| C_{1} C_{2} C .
\end{aligned}
$$


Similarly, we can get by (4.17)

$$
\begin{aligned}
& \mathbb{E}\left\|\int_{\tau_{1}}^{t} e^{(t-s)(\Delta-1)}\left[f_{i}(u(s, X))+v_{i}(t, X)+u_{i}(s, X)\right] d s\right\|_{L^{\infty}(\Omega)} \\
\leqslant & C_{1} C_{2} M \mathbb{E} \int_{\tau_{1}}^{t}(t-s)^{-\delta} e^{-\lambda_{2}(t-s)}\left\|f_{i}(u(s, X))\right\|_{L^{2}(\Omega)} d s \\
& +C_{1} C_{2} M \mathbb{E} \int_{\tau_{1}}^{t}(t-s)^{-\delta} e^{-\lambda_{2}(t-s)}\left(\left\|v_{i}(s, X)\right\|_{L^{2}(\Omega)}+\left\|u_{i}(s, X)\right\|_{L^{2}(\Omega)}\right) d s \\
\leqslant & C_{1} C_{2} C^{2}+C_{1} C_{2} C^{2}
\end{aligned}
$$

Since the constant $C$ is big enough, the condition (A1) yields

$$
\left.\left(\sum_{j=1}^{3} \varphi_{\sigma i j}\left(u_{i}(s, X), u_{i}(s-\tau(s), X)\right)\right)^{2} \leqslant C \sum_{i=1}^{3}\left[u_{i}^{2}(s, X)+u_{i}^{2}(s-\tau(s), X)\right)\right] .
$$

Moreover, since the constant $C$ is big enough, we can estimate

$$
\begin{aligned}
& \mathbb{E}\left\|\int_{\tau_{1}}^{t} e^{(t-s)(\Delta-1)} \sum_{j=1}^{3} \varphi_{\sigma i j}\left(u_{i}(s, X), u_{i}(s-\tau(s), X)\right) d w_{j}(s)\right\|_{L^{\infty}(\Omega)} \\
\leqslant & C \mathbb{E} \sqrt{\left.C_{1} \int_{\tau_{1}}^{t} \|(-\Delta+1)^{\delta} e^{2(t-s)(\Delta-1)} \sum_{i=1}^{3}\left[u_{i}^{2}(s, X)+u_{i}^{2}(s-\tau(s), X)\right)\right] \|_{L^{2}(\Omega)} d s} \\
\leqslant & C \sum_{i=1}^{3} \mathbb{E} \sqrt{\left.C_{1} C_{2} M \int_{\tau_{1}}^{t}(2 t-2 s)^{-\delta} e^{-2 \lambda_{2}(t-s)}\left[\left\|u_{i}^{2}(s, X)\right\|_{L^{2}(\Omega)}+\| u_{i}^{2}(s-\tau(s), X)\right) \|_{L^{2}(\Omega)}\right] d s} \\
\leqslant & C^{2} .
\end{aligned}
$$

Finally, combining (4.18)-(4.21) results in

$$
\left\|u_{i}(t, X)\right\|_{L^{\infty}(\Omega)} \leqslant C^{3}, i=1,2,3 .
$$

And then the proof is completed.

Now we can know from Remark 7 that Theorem 4.2 has been proved.

\section{Numerical example}

Example 5.1. Let $\mathfrak{T}=\{1,2\}$ in the system (2.18).

In the case $\sigma=1$,

$$
\left\{\begin{array}{l}
d X(t)=\left[H_{1} X(t)+f(X(t))+v(t)\right] d t+\varphi_{\sigma}(X(t), X(t-\tau(t))) d w(t), \quad t \geqslant 0 \\
X(s)=\xi(s), \quad s \in[-\tau, 0]
\end{array}\right.
$$


In the case $\sigma=2$,

$$
\left\{\begin{array}{l}
d X(t)=\left[H_{2} X(t)+f(X(t))+v(t)\right] d t+\varphi_{\sigma}(X(t), X(t-\tau(t))) d w(t), \quad t \geqslant 0 \\
X(s)=\xi(s), \quad s \in[-\tau, 0]
\end{array}\right.
$$

Let $a=2, b=0.4, c=2$, then

$$
H_{1}=\left(\begin{array}{ccc}
\frac{1}{c} & \theta & 1 \\
-2 \theta & -b & 0 \\
-1 & 0 & -c
\end{array}\right)=\left(\begin{array}{ccc}
0.5000 & 0 & 1.0000 \\
0 & -0.4000 & 0 \\
-1.0000 & 0 & -2.0000
\end{array}\right)=\left(\begin{array}{ccc}
\frac{1}{b}-a & 0 & 1 \\
0 & -b & 0 \\
-1 & 0 & -c
\end{array}\right)=H_{2} .
$$

Besides, set $\alpha_{1}=0.85, \varepsilon=0.5, \psi=0.001 I, \Lambda_{\sigma 1} \equiv 0.0001 I \equiv \Lambda_{\sigma 2}$, and

and

$$
\bar{D}_{1}=0, \bar{D}_{2}=\left(\begin{array}{ccc}
-2.5 & -1.5 & -1.2 \\
0 & 0 & 0 \\
0 & 0 & 0
\end{array}\right)
$$

Using the computer Matlab LMI toolbox to solve the LMI condition (3.2) results in the following feasible data:

$$
\beta_{1}=0.0107, \beta_{2}=0.9893, P=\left(\begin{array}{ccc}
0.9993 & 0 & 0 \\
0 & 0.9993 & 0 \\
0 & 0 & 0.9976
\end{array}\right) ; Q=\left(\begin{array}{ccc}
0.6873 & 0 & 0.0011 \\
0 & 0.5987 & 0.0001 \\
0.0011 & 0.0001 & 0.6676
\end{array}\right)
$$

Now, according to Theorem 3.1, the switched system (5.1) is said to be the global asymptotical input-to-state stability in the meaning of switching.

Example 5.2. Consider the following fuzzy switched financial system with $n=2$ (the number of the IF-THEN rules),

Fuzzy Rule 1: IF $\hat{\omega}_{1}(t)$ is $v_{11}$ and $\hat{\omega}_{2}(t)$ is $v_{12}$ THEN

$$
\left\{\begin{array}{l}
d X(t)=\left[H_{\sigma 1} X(t)+f(X(t))+v(t)\right] d t+\varphi_{\sigma}(X(t), X(t-\tau(t))) d w(t), \quad t \geqslant 0 \\
X(s)=\xi(s), \quad s \in[-\tau, 0]
\end{array}\right.
$$

In the case $r=1$ equipped with $a=0.9, b=0.63, c=1.5$, then

$$
\begin{aligned}
H_{11} & =\left(\begin{array}{ccc}
\frac{1}{c} & \theta & 1 \\
-2 \theta & -b & 0 \\
-1 & 0 & -c
\end{array}\right)=\left(\begin{array}{ccc}
0.6667 & 0.1140 & 1.0000 \\
-0.2280 & -0.6300 & 0 \\
-1.0000 & 0 & -1.5000
\end{array}\right) \\
H_{21} & =\left(\begin{array}{ccc}
\frac{1}{b}-a & 0 & 1 \\
0 & -b & 0 \\
-1 & 0 & -c
\end{array}\right)=\left(\begin{array}{ccc}
0.6873 & 0 & 1.0000 \\
0 & -0.6300 & 0 \\
-1.0000 & 0 & -1.5000
\end{array}\right)
\end{aligned}
$$




$$
H_{31}=\left(\begin{array}{ccc}
\frac{1}{c} & -\theta & 1 \\
2 \theta & -b & 0 \\
-1 & 0 & -c
\end{array}\right)=\left(\begin{array}{ccc}
0.6667 & -0.1140 & 1.0000 \\
0.2280 & -0.6300 & 0 \\
-1.0000 & 0 & -1.5000
\end{array}\right)
$$

Fuzzy Rule 2: IF $\hat{\omega}_{1}(t)$ is $v_{21}$ and $\hat{\omega}_{2}(t)$ is $v_{22}$ THEN

$$
\left\{\begin{array}{l}
d X(t)=\left[H_{\sigma 2} X(t)+f(X(t))+v(t)\right] d t+\varphi_{\sigma}(X(t), X(t-\tau(t))) d w(t), \quad t \geqslant 0 \\
X(s)=\xi(s), \quad s \in[-\tau, 0]
\end{array}\right.
$$

In the case $r=2$ equipped with $a=0.8, b=0.6, c=1.2$, then

$$
\begin{aligned}
& H_{12}=\left(\begin{array}{ccc}
0.8333 & 0.1414 & 1.0000 \\
-0.2828 & -0.6000 & 0 \\
-1.0000 & 0 & -1.2000
\end{array}\right) \\
& H_{22}=\left(\begin{array}{ccc}
0.8667 & 0 & 1.0000 \\
0 & -0.6000 & 0 \\
-1.0000 & 0 & -1.2000
\end{array}\right) \\
& H_{32}=\left(\begin{array}{ccc}
0.8333 & -0.1414 & 1.0000 \\
0.2828 & -0.6000 & 0 \\
-1.0000 & 0 & -1.2000
\end{array}\right)
\end{aligned}
$$

Set

$$
\begin{gathered}
E_{1}=\left(\begin{array}{ccc}
0.1000 & 0.06385 & 0 \\
0.06385 & 0.0500 & 0 \\
0 & 0 & 0.3000
\end{array}\right), E_{2}=\left(\begin{array}{ccc}
0.0900 & 0 & 0 \\
0 & 0.0160 & 0 \\
0 & 0 & 0.1600
\end{array}\right) E_{3}=\left(\begin{array}{ccc}
0.1000 & -0.06385 & 0 \\
-0.06385 & 0.1150 & 0 \\
0 & 0 & 0.3000
\end{array}\right) \\
\bar{D}_{1}=0=\bar{D}_{3}, \bar{D}_{2}=\left(\begin{array}{ccc}
-1.0544 & 0 & 0 \\
0 & 0 & 0 \\
0 & 0 & 0
\end{array}\right)
\end{gathered}
$$


Due to $0 \leqslant \varrho_{r}(\hat{\omega}(t)) \leqslant 1$ and $\sum_{r=1}^{2} \varrho_{r}(\hat{\omega}(t))=1$, we can get by computing

$$
\begin{aligned}
& \left.\sum_{r=1}^{2} \varrho_{r}(\hat{\omega}(t))\left(H_{1 r}+H_{1 r}^{T}\right)=\varrho_{1}(\hat{\omega}(t))\left(H_{11}+H_{11}^{T}\right)+\varrho_{2}(\hat{\omega}(t))\left(H_{12}+H_{12}^{T}\right)\right] \\
& =\varrho_{1}(\hat{\omega}(t))\left(\begin{array}{ccc}
1.3333 & -0.1140 & 0 \\
-0.1140 & -1.2600 & 0 \\
0 & 0 & -3.0000
\end{array}\right)+\varrho_{2}(\hat{\omega}(t))\left(\begin{array}{ccc}
1.6667 & -0.1414 & 0 \\
-0.1414 & -1.2000 & 0 \\
0 & 0 & -2.4000
\end{array}\right) \\
& \leqslant \max _{s \in[0,1]}\left[s\left(\begin{array}{ccc}
1.3333 & -0.1140 & 0 \\
-0.1140 & -1.2600 & 0 \\
0 & 0 & -3.0000
\end{array}\right)+(1-s)\left(\begin{array}{ccc}
1.6667 & -0.1414 & 0 \\
-0.1414 & -1.2000 & 0 \\
0 & 0 & -2.4000
\end{array}\right)\right] \\
& <\left(\begin{array}{ccc}
1.7000 & 0 & 0 \\
0 & -1.1300 & 0 \\
0 & 0 & -2.1000
\end{array}\right) \\
& =\left(\begin{array}{ccc}
1.5000 & -0.1277 & 0 \\
-0.1277 & -1.2300 & 0 \\
0 & 0 & -2.7000
\end{array}\right)+2\left(\begin{array}{ccc}
0.1 & 0.06385 & 0 \\
0.06385 & 0.0500 & 0 \\
0 & 0 & 0.3
\end{array}\right) \\
& =\frac{1}{2} \sum_{r=1}^{2}\left(H_{1 r}+H_{1 r}^{T}\right)+E_{1}+E_{1}^{T}
\end{aligned}
$$


Similarly, we get

$$
\begin{aligned}
& \sum_{r=1}^{2} \varrho_{r}(\hat{\omega}(t))\left(H_{2 r}+H_{2 r}^{T}\right)=\varrho_{1}(\hat{\omega}(t))\left(H_{21}+H_{21}^{T}\right)+\varrho_{2}(\hat{\omega}(t))\left(H_{22}+H_{22}^{T}\right) \\
& =\varrho_{1}(\hat{\omega}(t))\left(\begin{array}{ccc}
1.3746 & 0 & 0 \\
0 & -1.2600 & 0 \\
0 & 0 & -3.0000
\end{array}\right)+\varrho_{2}(\hat{\omega}(t))\left(\begin{array}{ccc}
1.7333 & 0 & 0 \\
0 & -1.2000 & 0 \\
0 & 0 & -2.4000
\end{array}\right) \\
& \leqslant\left(\begin{array}{ccc}
1.7333 & 0 & 0 \\
0 & -1.2000 & 0 \\
0 & 0 & -2.4000
\end{array}\right) \\
& <\left(\begin{array}{ccc}
1.7339 & 0 & 0 \\
0 & -1.1980 & 0 \\
0 & 0 & -2.3800
\end{array}\right) \\
& =\left(\begin{array}{ccc}
1.5539 & 0 & 0 \\
0 & -1.2300 & 0 \\
0 & 0 & -2.7000
\end{array}\right)+2\left(\begin{array}{ccc}
0.0900 & 0 & 0 \\
0 & 0.0160 & 0 \\
0 & 0 & 0.1600
\end{array}\right) \\
& =\frac{1}{2} \sum_{r=1}^{2}\left(H_{2 r}+H_{2 r}^{T}\right)+E_{2}+E_{2}^{T}
\end{aligned}
$$

and

$$
\begin{aligned}
& \left.\sum_{r=1}^{2} \varrho_{r}(\hat{\omega}(t))\left(H_{3 r}+H_{3 r}^{T}\right)=\varrho_{1}(\hat{\omega}(t))\left(H_{31}+H_{31}^{T}\right)+\varrho_{2}(\hat{\omega}(t))\left(H_{32}+H_{32}^{T}\right)\right] \\
& =\varrho_{1}(\hat{\omega}(t))\left(\begin{array}{ccc}
1.3333 & 0.1140 & 0 \\
0.1140 & -1.2600 & 0 \\
0 & 0 & -3.0000
\end{array}\right)+\varrho_{2}(\hat{\omega}(t))\left(\begin{array}{ccc}
1.6667 & 0.1414 & 0 \\
0.1414 & -1.2000 & 0 \\
0 & 0 & -2.4000
\end{array}\right) \\
& \leqslant \max _{s \in[0,1]}\left[s\left(\begin{array}{ccc}
1.3333 & 0.1140 & 0 \\
0.1140 & -1.2600 & 0 \\
0 & 0 & -3.0000
\end{array}\right)+(1-s)\left(\begin{array}{ccc}
1.6667 & 0.1414 & 0 \\
0.1414 & -1.2000 & 0 \\
0 & 0 & -2.4000
\end{array}\right)\right] \\
& <\left(\begin{array}{ccc}
1.7000 & 0 & 0 \\
0 & -1.0000 & 0 \\
0 & 0 & -2.1000
\end{array}\right) \\
& =\left(\begin{array}{ccc}
1.5000 & 0.1277 & 0 \\
0.1277 & -1.2300 & 0 \\
0 & 0 & -2.7000
\end{array}\right)+2\left(\begin{array}{ccc}
0.1000 & -0.06385 & 0 \\
-0.06385 & 0.115 & 0 \\
0 & 0 & 0.3000
\end{array}\right) \\
& =\frac{1}{2} \sum_{r=1}^{2}\left(H_{3 r}+H_{3 r}^{T}\right)+E_{3}+E_{3}^{T} \text {. }
\end{aligned}
$$


Combining (5.3)-(5.5) means that the fuzzy estimate condition (A2) is satisfied.

Set

$$
\varphi_{\sigma}(X(t), X(t-\tau(t))) \equiv\left(\begin{array}{ccc}
0.15 X_{1}(t-\tau(t)) & 0 & 0 \\
0 & 0.15 X_{2}(t-\tau(t)) & 0 \\
0 & 0 & 0.13 X_{3}(t-\tau(t))
\end{array}\right)
$$

then we let

$$
\Lambda_{\sigma 1} \equiv 0.0001 I \equiv \Lambda_{\sigma 2}, \quad \alpha_{\sigma} \equiv 0.03
$$

which implies that the condition (A2) holds.

Let $\varsigma=0.05, q=1.2, \tau=5$, and then employing computer Matlab LMI toolbox to solve (3.1) results in

$$
\beta_{1}=0.0150, \beta_{2}=0.9750, \beta_{3}=0.0100, \quad \Psi=\left(\begin{array}{ccc}
0.01856 & 0 & 0 \\
0 & 0.01396 & 0 \\
0 & 0 & 0.01766
\end{array}\right)>0
$$

According to Theorem 3.1, the fuzzy switched system (5.2) is exponential input-to-state stability in the meaning of switching.

\section{Conclusions}

Although the non-Lipschitz functions make the free weight matrix technique unsuitable for financial systems, in this paper, LMI-based criterion of globally asymptotical stability in the meaning of switching for chaos delayed feedback switched stochastic financial system was obtained, and the globally asymptotical stability in the meaning of switching is more suitable for the financial system, which implies that when the time $t$ is big enough, the dynamic of any subsystem must approach its unique equilibrium point. Besides, the global financial crisis often erupts periodically, which illuminates that the global stability in the classical sense is actually meaningless. In addition, boundedness result and the exponential stability criterion was also derived for fuzzy financial system in the meaning of switching. Numerical examples shows the effectiveness of the proposed methods.

\section{Funding Statement}

The work is supported by the Application basic research project of science and Technology Department of Sichuan Province (No. 2020YJ0434) and the Major scientific research projects of Chengdu Normal University in 2019 (No. CS19ZDZ01)

\section{References}


[1] Chengdai Huang, Liming Cai, Jinde Cao. Linear control for synchronization of a fractional-order time-delayed chaotic financial system. Solitons \& Fractals, Volume 113, August 2018, Pages 326-332

[2] Gao, Q., Ma, J.: Chaos and Hopf bifurcation of a finance system. Nonlinear Dynamics, 58, 209 (2009). https://doi.org/10.1007/s11071-0099472-5

[3] Xiao-Li Gong, Xi-Hua Liu, Xiong Xiong. Chaotic analysis and adaptive synchronization for a class of fractional order financial system. Physica A: Statistical Mechanics and its Applications, 2019, 522, 33-42.

[4] Zhao M., Wang J. $H_{\infty}$ control of a chaotic finance system in the presence of external disturbance and input time-delay. Appl. Math. Comput. 233, 320-327 (2014)

[5] Yanli Cao. Chaotic synchronization based on fractional order calculus financial system. Chaos, Solitons \& Fractals, Volume 130, January 2020, 109410

[6] Ahmad Hajipour, Hamidreza Tavakoli. Dynamic Analysis and Adaptive Sliding Mode Controller for a Chaotic Fractional Incommensurate Order Financial System. International Journal of Bifurcation and Chaos, Vol. 27, No. 13, 1750198 (2017) .

[7] Ruofeng Rao, Shouming Zhong. Impulsive control on delayed feedback chaotic financial system with Markovian jumping. Advances in Difference Equations, 2020, 2020:50

[8] Rao, R., Zhong, S.: Input-to-state stability and no-inputs stabilization of delayed feedback chaotic financial system involved in open and closed economy. Discrete and Continuous Dynamical Systems ( Series S ) 1-19 (2020). https://doi.org/10.3934/dcdss.2020280

[9] Rao R. Global Stability of a Markovian Jumping Chaotic Financial System with Partially Unknown Transition Rates under Impulsive Control Involved in the Positive Interest Rate. Mathematics, 7(7), 579 (2019) https://doi.org/10.3390/math7070579

[10] Jing Duan. Financial system modeling using deep neural networks (DNNs) for effective risk assessment and prediction. Journal of the Franklin Institute, Volume 356, Issue 8May 2019Pages 4716-4731

[11] Qiankun Song, Jinde Cao. Dynamical behaviors of discrete-time fuzzy cellular neural networks with variable delays and impulses. Journal of the Franklin Institute, Volume 345, Issue 1January 2008Pages 39-59

[12] Dianhao Zheng, Hongbin Zhang, J. Andrew Zhang, Weixing Zheng, Steven W. Su. Stability of asynchronous switched systems with sequence-based average dwell time approaches. Journal of the Franklin Institute, Volume 357, Issue 4March 2020Pages 2149-2166

[13] R. Sakthivel, S. Selvi, K. Mathiyalagan, P. Shi, Reliable mixed Hand passivity-based control for fuzzy markovian switching systems with probabilistic time delays and actuator failures, IEEE Trans. Cybern. 23(3) (2015) 2720-2731.

[14] T. Takagi, M. Sugeno, Fuzzy identification of systems and its application to modeling and control, IEEE Trans. Syst. Man Cybern. Syst. $15(2.1)(1985)$ 116-132.

[15] Qiankun Song, Zhenjiang Zhao, Jianxi Yang. Passivity and passification for stochastic Takagi-Sugeno fuzzy systems with mixed time-varying delays Neurocomputing Volume 12225 December 2013Pages 330-337

[16] Z.P. Wang, H.N. Wu, Fuzzy impulsive control for uncertain nonlinear systems with guaranteed cost, Fuzzy Sets Syst. 302 (2016) 143-162.

[17] Qiankun Song, Zidong Wang. Dynamical behaviors of fuzzy reaction-diffusion periodic cellular neural networks with variable coefficients and delays Applied Mathematical ModellingVolume 33, Issue 9September 2009Pages 3533-3545

[18] Shujun Long, Qiankun Song, Xiaohu Wang, Dingshi Li. Stability analysis of fuzzy cellular neural networks with time delay in the leakage term and impulsive perturbations. Journal of the Franklin Institute, Volume 349, Issue 7September 2012Pages 2461-2479

[19] Dan Yang, Xiaodi Li, Shiji Song. Design of State-Dependent Switching Laws for Stability of Switched Stochastic Neural Networks With Time-Delays. IEEE Transactions on Neural Networks and Learning Systems, 2019.

[20] J. Cheng, J.H. Park, Y.J. Liu, Z.J. Liu, L.M. Tang, Finite-time Hfuzzy control of nonlinear Markovian jump delayed systems with partly uncertain transition descriptions, Fuzzy Sets Syst. 314 (2017) 99-115.

[21] Jiaojiao Ren, Xinzhi Liu, Hong Zhu, Shouming Zhong, Cong Wu. Exponential H synchronization of switching fuzzy systems with timevarying delay and impulses. Fuzzy Sets and SystemsVolume 36515 June 2019Pages 116-139

[22] Qian Li, Xinzhi Liu, Qingxin Zhu, Shouming Zhong, Dian Zhang. Distributed state estimation for stochastic discrete-time sensor networks with redundant channels. Applied Mathematics and ComputationVolume 34315 February 2019Pages 230-246 
[23] Shiyu Dong, Hong Zhu, Shouming Zhong, Kaibo Shi, Wei Kang. New result on reliable H performance state estimation for memory static neural networks with stochastic sampled-data communication Applied Mathematics and Computation, Volume 3641 January 2020Article 124619

[24] Mengzhuo Luo, Xinzhi Liu, Shouming Zhong, Jun Cheng. Synchronization of multi-stochastic-link complex networks via aperiodically intermittent control with two different switched periods Physica A: Statistical Mechanics and its Applications Volume 5091 November 2018Pages 20-38

[25] Xiaodi Li, Xilin Fu. Stability analysis of stochastic functional differential equations with infinite delay and its application to recurrent neural networks. Journal of Computational and Applied Mathematics 234 (2010) 407-417.

[26] Ruofeng Rao, Shouming Zhong, Zhilin Pu. Fixed point and p-stability of T-S fuzzy impulsive reaction-diffusion dynamic neural networks with distributed delay via Laplacian semigroup NeurocomputingVolume 33528 March 2019Pages 170-184

[27] Hong Cheng, Shouming Zhong, Xiaoqing Li, Qishui Zhong, Jun Cheng. Exponential synchronization of delayed memristor-based neural networks with stochastic perturbation via nonlinear control. Neurocomputing, Volume 3407 May 2019Pages 90-98

[28] D. Henry, Geometric Theory of Semilinear Parabolic Equations, Lecture Notes Math., 840, Springer-Verlag, Berlin, New York, 1981.

[29] D. Horstmann, M. Winkler, Boundedness vs. blow-up in a chemotaxis system, J. Diff. Equ. 215 (2005), 52-107.

[30] Ruofeng Rao. Delay-Dependent Exponential Stability for Nonlinear Reaction-Diffusion Uncertain Cohen-Grossberg Neural Networks with Partially Known Transition Rates via Hardy-Poincare Inequality. Chinese Annals of Mathematics(Series B),2014,35(04):575-598.

[31] Ruofeng Rao, Shouming Zhong, Xiongrui Wang. Stochastic stability criteria with LMI conditions for Markovian jumping impulsive BAM neural networks with mode-dependent time-varying delays and nonlinear reaction-diffusion. Communications in Nonlinear Science and Numerical Simulation, 2014, 19, 258-273.

[32] Ruofeng Rao. Stability Analysis of Nontrivial Stationary Solution and Constant Equilibrium Point of Reaction-Diffusion Neural Networks with Time Delays under Dirichlet Zero Boundary Value. Preprints 2020, 2020040277 (doi: 10.20944/preprints202004.0277.v3).

[33] X. Mao, Stochastic Differential Equations and Applications, Horwood, 1997.

[34] Daoyi Xu, Zhiguo Yang, Yumei Huang. Existence-uniqueness and continuation theorems for stochastic functional differential equations. J. Differential Equations, 245 (2008) 1681-1703

[35] Wenjuan Xie, Quanxin Zhu. Input-to-state stability of stochastic nonlinear fuzzy Cohen-Grossberg neural networks with the event-triggered control. International Journal of Control, 2020, 93(9):2043-2052.

[36] Xiaodi Li, Xiaoli Zhang, Shiji Song. Effect of delayed impulses on input-to-state stability of nonlinear systems, Automatica, 2017, 76, 378-382

[37] Xiaodi Li, Peng Li, Qing-guo Wang. Input/output-to-state stability of impulsive switched systems Systems \& Control Letters, 116, 2018, $1-7$.

[38] Peng Li, Xiaodi Li. Input-to-state stability of nonlinear impulsive systems via Lyapunov method involving indefinite derivative Mathematics and Computers in Simulation, 2019,155, 314-323

[39] Haitao Zhu, Peng Li, Xiaodi Li, Haydar Akca. Input-to-state stability for impulsive switched systems with incommensurate impulsive switching signals. Communications in Nonlinear Science and Numerical Simulation, 2020, 80, 104969 